\title{
Turning ZnO into an Efficient Energy Upconversion Material by Defect Engineering
}

Jan Eric Stehr, Shula Chen, Nandanapalli Koteeswara Reddy, Charles W. Tu, Weimin Chen and Irina Buyanova

\section{Linköping University Post Print}

\section{Tweet}

N.B.: When citing this work, cite the original article.

Original Publication:

Jan Eric Stehr, Shula Chen, Nandanapalli Koteeswara Reddy, Charles W. Tu, Weimin Chen and Irina Buyanova, Turning ZnO into an Efficient Energy Upconversion Material by Defect Engineering, 2014, Advanced Functional Materials, (24), 24, 3760-3764.

http://dx.doi.org/10.1002/adfm.201400220

Copyright: Wiley-VCH Verlag http://www.wiley-vch.de/publish/en/

Postprint available at: Linköping University Electronic Press http://urn.kb.se/resolve?urn=urn:nbn:se:liu:diva-109192 
DOI: $10.1002 / \mathrm{adfm} .201400220$

Article type: Full paper

\title{
Turning $\mathrm{ZnO}$ into an efficient energy upconversion material by defect engineering
}

\author{
Jan E. Stehr, Shula L. Chen, Nandanapalli Koteeswara Reddy, Charles W. Tu, \\ Weimin M. Chen, and Irina A. Buyanova*
}

Dr. J. E. Stehr, S. L. Chen, Prof. W. M. Chen, Prof. I. A. Buyanova

Department of Physics, Chemistry and Biology, Linköping University, 58183 Linköping, Sweden

E-mail: iribu@ifm.liu.se

Dr. N. Koteeswara Reddy

Department of Nanobio Materials and Electronics, Gwangju Institute of Science and Technology, Gwangju 500712, Republic of Korea

Prof. C. W. Tu

Department of Electrical and Computer Engineering, University of California, La Jolla, CA 92093, USA

Keywords: Nonlinear Optics, Zinc Oxide, Zinc Vacancy, Photoluminescence, Electron Paramagnetic Resonance

\begin{abstract}
Photon upconversion materials are attractive for a wide range of applications from medicine, biology to photonics. Among them, $\mathrm{ZnO}$ is of particular interest owing to its outstanding combination of materials and physical properties. Though energy upconversion has been demonstrated in $\mathrm{ZnO}$, the exact physical mechanism is still unknown that prevents a control of the processes. Here, we show that defects formed in bulk and nanostructured $\mathrm{ZnO}$ synthesized using standard growth techniques play a key role in promoting efficient energy upconversion via two-step two-photon absorption (TS-TPA). From photoluminescence excitation of the anti-Stokes emissions, the threshold energy of the TS-TPA process is determined as being $2.10-2.14 \mathrm{eV}$ in all studied $\mathrm{ZnO}$ materials irrespective of the employed growth techniques. Our photo- electron paramagnetic resonance studies show that this threshold closely matches the ionization energy of the zinc vacancy - a common grown-in intrinsic defect in $\mathrm{ZnO}$, thereby identifying the zinc vacancy as being the dominant defect responsible for the observed efficient energy upconversion. The upconversion is found to persist even at a low excitation density, making it attractive for photonic and photovoltaic applications.
\end{abstract}




\section{Introduction}

$\mathrm{ZnO}$ is a II-VI semiconductor that holds a great promise for a variety of photonic applications owing to its wide and direct bandgap and a large exciton binding energy, combined with established growth processes that allow fabrication of $\mathrm{ZnO}$ with superior quality in a variety of morphologies ranging from bulk to nanostructures. ${ }^{[1,2]} \mathrm{As}$ a result, $\mathrm{ZnO}$ is currently considered among the key materials for UV light emitting devices, solid-state white lighting, transparent conductive oxides and UV photovoltaic cells with tailored dimensions. ${ }^{[3]}$ Furthermore, as a bio-compatible material, $\mathrm{ZnO}$ is attractive for biophotonics and bio-medical applications including cell and tissue imaging. ${ }^{[4]}$

Recently it was reported ${ }^{[5-7]}$ that $\mathrm{ZnO}$ can also exhibit efficient photon upconversion, i. e. capable of converting two low-energy photons into a single higher-energy photon, which could open a window of opportunities for improved and even new device applications. Indeed energy upconversion currently attracts great attention in diverse research fields ranging from medicine and biology to optoelectronics and photonics. ${ }^{[5,8-15]}$ In biological labeling and imaging, upconversion of photoluminescence (PL) allows improved sensitivity, an increased penetration depth and spatially resolved imaging of biological objects. ${ }^{\left[{ }^{[9}\right.}$ In light emitting devices, it can be used for white light generation ${ }^{[10]}$ and can also provide a new way for laser pumping, ${ }^{[5,11]}$ representing a viable alternative to frequency-doubled coherent light sources. For example, by using $\mathrm{ZnO}$ it allows one to replace expensive UV pumping sources by existing, inexpensive visible/near-infrared laser diodes based on III-V semiconductors. This will be found advantageous for low-cost implementation of $\mathrm{ZnO}$ nanolasers in photonic circuits. ${ }^{[5]}$ In photovoltaic devices, energy upconversion allows to increase efficiency of sunlight harvesting beyond the Shockley-Queisser limit by consumption of photons with energies below the bandgap of a light absorber. ${ }^{[12]}$ Two-photon absorption was also suggested to be a potential method for information processing in three-dimensional optical storage 
memory ${ }^{[13]}$ and has been considered as a key process in volumetric display technology. ${ }^{[14]} \mathrm{We}$ should also note that whereas energy upconversion is desirable for many applications, it may also become an obstacle when using $\mathrm{ZnO}$ as a transparent conductor as this process will decrease the transparency of the material for visible light.

Light upconversion in $\mathrm{ZnO}$ can proceed via several processes including two-photon absorption (TPA) and two-step two-photon absorption (TS-TPA) ${ }^{[5-7]}$. In both processes excitation of carriers is accomplished by sequential absorption of two photons via an intermediate state. TPA occurs via a virtual state and thus requires rather high excitation densities (i.e. of the order of $\mathrm{P}_{\mathrm{exc}}=10^{9} \mathrm{~W} \mathrm{~cm}^{-2}$ ) in spite of a lack of inversion symmetry adherent to the wurtzite crystal structure of this material. On the other hand, TS-TPA can be achieved at remarkably low $\mathrm{P}_{\mathrm{exc}} \sim 0.1 \mathrm{~W} \mathrm{~cm}^{-2}{ }^{[7]}$ owing to the fact that the intermediate state involved in this process is a real state, e.g. an energy level of a defect or impurity. This process therefore dominates in low excitation density regimes and has the benefit of relatively high (i.e. in the order of $1-2 \%$ at low $\mathrm{P}_{\mathrm{exc}}$ ) conversion efficiency without the need for intense excitation sources.

Optimization of the TS-TPA process requires in-depth understanding of material properties so that defects/impurities which introduce the intermediate state are first reliably identified and then deliberately incorporated in (or removed from) the material. Such understanding, however, is currently lacking. The key questions, i.e. what is the origin of the defect/impurity responsible for the TS-TPA process in $\mathrm{ZnO}$ and the exact energy position of the corresponding intermediate state, remain unanswered, though we have most recently suggested $^{[7]}$ that a native defect may be involved. The purpose of the present work is to reliably answer these questions based on comprehensive electron paramagnetic resonance (EPR) studies combined with detailed spectral dependent measurements of TS-TPA. The EPR spectroscopy is chosen in this study as this technique, together with its optical counterpart optically detected magnetic resonance (ODMR), is among the most powerful and versatile 
experimental methods for defect identification. It has been used in the past to provide chemical identity and local structure of various intrinsic defects and impurities in $\mathrm{ZnO}$, and also to determine their energy level positions within the bandgap. ${ }^{[16,17]}$ Furthermore, knowledge gained from spectral dependence of TS-TPA based on excitation spectroscopy of upconverted PL will allow us to accurately determine the energy position of the intermediate level involved in the upconversion, which could then be correlated with the results of EPR studies to uncover the defect identity.

\section{Results and Discussion}

In order to evaluate whether the TS-TPA process in $\mathrm{ZnO}$ has a common origin, several types of $\mathrm{ZnO}$ synthesized using standard growth techniques were selected for this study. These include commercially available melt-grown and hydrothermally-grown bulk substrates and $\mathrm{ZnO}$ nanowires (NWs) grown by rapid thermal chemical vapor deposition on c-plane $\mathrm{Al}_{2} \mathrm{O}_{3}$ substrates. Light upconversion was monitored by the intensity of donor bound exciton (BX) emissions.

All investigated structures exhibit energy upconversion, obvious from the appearance of intense near-band-edge emissions due to BX recombination under anti-Stokes excitation by visible light. This is demonstrated in Figure 1, which shows representative PL spectra measured with $\lambda_{\text {exc }}=400 \mathrm{~nm}$ (the solid lines). The spectra of the bulk materials (Figure 1(a) and $1(\mathrm{~b})$ ) contain well-known BX lines labeled as $\mathrm{I}_{9}, \mathrm{I}_{7}, \mathrm{I}_{6}, \mathrm{I}_{4}$ and $\mathrm{I}^{*}$ adopting the notations from Reference 18 and 19. Some of these lines (e.g. $I_{9}$, and $\mathrm{I}_{7}$ ) are also likely detected in the $\mathrm{ZnO}$ NWs, though broadened and shifted by about $0.5 \mathrm{meV}$ relative to their energy positions in bulk $\mathrm{ZnO}$. These lines are labeled in Figure $1(\mathrm{c})$ as $\mathrm{DX}_{1}$ and $\mathrm{DX}_{2}$, respectively. All these spectra are very similar to those measured under conventional one-photon excitation with $\lambda_{\mathrm{exc}}$ $=266 \mathrm{~nm}$ above the $\mathrm{ZnO}$ bandgap energy (shown by the dashed lines in Figure 1). The 
relative contributions of different excitonic emissions vary between the one- and two-photon excitation conditions, however, likely due to strong re-absorption effects that become important in the latter case.

The observed energy upconversion can be accomplished within a wide spectral range of 380 $735 \mathrm{~nm}$, due to combined effects of TPA and TS-TPA. Contributions of these processes can be differentiated by measuring dependence of the upconverted PL (UPL) intensity, IUPL, on

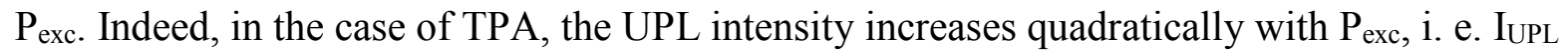
$\sim \mathrm{P}^{\mathrm{n}}$ exc with $\mathrm{n}=2$, whereas a weaker power dependence with $\mathrm{n}<2$ is typical for TS-TPA via a long-lived real state. Our detailed power-dependent measurements of the studied structures using light sources with a fixed wavelength ${ }^{[7]}$ have shown that TPA can be detected for all photons with wavelengths between 380 and $735 \mathrm{~nm}$ but prevails at high $\mathrm{P}_{\mathrm{exc}}>10 \mathrm{~W} \mathrm{~cm}{ }^{-2}$. It is also the only process responsible for the UPL excitation for $\lambda_{\mathrm{exc}}=661-735 \mathrm{~nm}$. On the other hand, TS-TPA allows efficient energy upconversion in the very weak excitation regime (i.e. for $\mathrm{P}_{\mathrm{exc}}$ as low as $0.1-10 \mathrm{~W} \mathrm{~cm}^{-2}$ ) and has a spectral threshold somewhere between 532 and $661 \mathrm{~nm}$.

To determine the exact energy position of the involved intermediate state, we have performed PLE measurements using a tunable dye-laser. Figure 2 shows the dependence of the BX UPL intensity divided by $\mathrm{P}^{2}$ exc measured from the bulk (a) and NW (b) ZnO. Presenting data in these coordinates allows us to precisely determine the spectral threshold of the TS-TPA process, as the IUPL $/ \mathrm{P}^{2}$ exc ratio is expected to remain constant when the TPA process dominates. The latter is indeed the case when $\lambda_{\text {exc }}$ exceeds $590 \mathrm{~nm}$, which proves that the energy upconversion under these conditions occurs solely via TPA. On the other hand, a steep rise of the UPL efficiency is observed when $\lambda_{\text {exc }}$ is tuned shorter than $590 \mathrm{~nm}$. This suggests activation of an additional upconversion process via TS-TPA as shown schematically in Figure 2(c). This assignment was further confirmed from excitation power dependent measurements performed for several $\lambda_{\text {exc }}$ in the vicinity of the threshold. Taking as an 
example the bulk $\mathrm{ZnO}$, the corresponding results for $\lambda_{\mathrm{exc}}=575$ and $610 \mathrm{~nm}$ are shown in Figure 2(d) by the filled circles and open squares, respectively. A best fit to the experimental data using the power-law function yields $\mathrm{n}=1.7$ and 2 for $\lambda_{\mathrm{exc}}=575$ and $610 \mathrm{~nm}$, respectively. The observed decrease in the power-law factor $\mathrm{n}$ for $\lambda_{\mathrm{exc}}<590 \mathrm{~nm}$ further proves that the energy upconversion now involves TS-TPA. It also implies that the energy level of the defect/impurity responsible for the two-photon absorption is located at $2.10-2.14 \mathrm{eV}$ below the conduction band or above the valence band. As also seen from Figure 2 this threshold energy is the same for all studied $\mathrm{ZnO}$ materials irrespective of the employed growth methods. This finding suggests that the TS-TPA process in $\mathrm{ZnO}$ is promoted by the same defect/impurity that is commonly present in $\mathrm{ZnO}$.

In order to identify the chemical origin of the responsible defect/impurity, we performed photo-EPR experiments. This is because a unique set of spin-Hamiltonian parameters determined from photo-EPR for each defect/impurity can be used as a fingerprint of the particular defect/impurity. Furthermore, an EPR signal can only be detected when the studied center is in a paramagnetic charge state with an unpaired electron spin. In dark, the charge state of the center is determined by the Fermi level position in the sample. The charge state can, however, be changed upon light illumination, provided that the photon energy is sufficient for photo-ionization of the involved defect/impurity. This makes possible to use photo-EPR measurements to determine energy level positions of various defects present in the samples and then to correlate them with the spectral threshold for the TS-TPA process. EPR results were analysed with the help of Easyspin ${ }^{[20]}$ using the following spin Hamiltonian:

$$
\mathrm{H}=\mu_{B} \boldsymbol{B g} \boldsymbol{S}+\boldsymbol{S D S}+\boldsymbol{S A I}
$$

Here the first term denotes the electronic Zeeman energy, with the Bohr magneton $\mu_{\mathrm{B}}$, the external magnetic field $\mathbf{B}$, the electron spin $\mathbf{S}$, and the electron $\mathrm{g}$-tensor $\mathbf{g}$. The fine structure splitting for $S>1 / 2$ is given by the second term with its interaction tensor $\mathbf{D}$. The third term describes the hyperfine interaction involving the nuclear spin I, quantified by the hyperfine 
tensor $\mathbf{A}$. The terms representing nuclear quadrupole and nuclear Zeeman interactions are omitted from Equation (1) as they play a negligible role in EPR spectra, except that the former may partially relax the selection rule of EPR transitions by admixing of nuclear spin states when I $>1 / 2$. The spin-Hamiltonian parameters of all detected EPR signals are summarized in Table I. These include common contaminants, such as $\mathrm{Mn}, \mathrm{Fe}, \mathrm{Li}, \mathrm{Al}$ and $\mathrm{Ga}$ impurities, as well as intrinsic defects such as oxygen $\left(V_{O}\right)$ and zinc $\left(V_{Z n}\right)$ vacancies.

Contributions of the aforementioned EPR signals were found to be material-dependent and were also affected by experimental conditions, i.e. light illumination and photon wavelength. To demonstrate these effects, Figure 3(a) shows representative EPR spectra measured at $77 \mathrm{~K}$ in dark and under light illumination, taking as an example the melt-grown bulk $\mathrm{ZnO}$. In dark, the EPR spectrum contains two signals. The first one, which is centered at $343.5 \mathrm{mT}$ with a line width of approximately $1 \mathrm{mT}$, is denoted by EM and originates from a shallow effective mass donor, likely an $\mathrm{Al}$ and/or a Ga impurity. The second signal spans over a wide range of magnetic fields (210 - $460 \mathrm{mT})$ and consists of five groups of six lines, which clearly indicates involvement of a paramagnetic center with $S=5 / 2$ and $I=5 / 2$. This signal is an EPR signature of a $\mathrm{Mn}^{2+}$ ion. ${ }^{[21]}$ Illumination at the wavelength of $610 \mathrm{~nm}$ does not change the EPR spectra. However, when the light wavelength is decreased to $600 \mathrm{~nm}$ an EPR signal from the positively changed $\mathrm{VO}^{+}$center ${ }^{[22]}$ is activated as a result of the light-induced photoionization which converts $V_{O}$ from the neutral to positively charged state:

$$
V_{O}^{0}+h v \rightarrow V_{O}^{+}+e
$$

This places the $(0 /+)$ level of this defect at around $2.0 \mathrm{eV}$ below the conduction band, consistent with previous photo-EPR studies. ${ }^{[23]}$ At an even shorter wavelength of $580 \mathrm{~nm}$, another EPR signal appears at $335.6 \mathrm{mT}$. Based on the determined spin-Hamiltonian parameters, this signal can be attributed to the negatively charged axial $\mathrm{VZn}^{-} .^{[24]}$ The corresponding photo-ionization process, 


$$
V_{Z n}^{2-}+h v \rightarrow V_{Z n}^{-}+e
$$

has a threshold at around $2.1 \mathrm{eV}$ in agreement with previously reported data. ${ }^{[1,23,24]} \mathrm{In}$ addition to $\mathrm{Mn}^{2+}, \mathrm{V}_{\mathrm{O}}^{+}$and $\mathrm{V}_{\mathrm{Zn}^{-}}$that dominate in the melt-grown $\mathrm{ZnO}$ discussed above, hydrothermally grown materials were found to contain Fe and Li that give rise to EPR signals arising from a neutral $\mathrm{Li}$ acceptor, $\mathrm{Li}_{\mathrm{Zn}}{ }^{0}{ }^{[25]}$ and a positive $\mathrm{Fe}^{3+}$ ion located at a $\mathrm{Zn}$ site. The $\mathrm{Lizn}^{0}$ was only observed under illumination with $\lambda_{\mathrm{exc}}<650 \mathrm{~nm}$, whereas the $\mathrm{Fe}^{3+}$ signal could also be detected in dark. Other common trace impurities, such as $\mathrm{Ni}, \mathrm{Cu}$ and $\mathrm{Pb}$ that are known to introduce deep levels in the bandgap of $\mathrm{ZnO},{ }^{[16,26]}$ were not present in sufficient concentrations to be detected by photo-EPR and thus to contribute to the observed TS-TPA. The illumination wavelength dependences of the EPR signals measured from the investigated bulk $\mathrm{ZnO}$ samples are summarized in Figure 3(b). It is obvious that though several defects and impurities participate in the light absorption within the visible spectral range, only $\mathrm{V}_{\mathrm{Zn}}$ has the energy level that exactly matches the spectral threshold of the TS-TPA deduced from the PLE measurements shown in Figure 3(b). This provides compelling evidence that the zinc vacancy is the dominant defect responsible for the observed TS-TPA process in bulk $\mathrm{ZnO}$. Moreover, an increase in the upconversion efficiency was observed for materials with a higher $\mathrm{V}_{\mathrm{Zn}}$ concentration, which further supports this conclusion.

From the PLE measurements (Figure 2), the same defects are also expected to be responsible for the TS-TPA in ZnO NWs. Unfortunately, the total number of the zinc vacancies was found to be below the EPR detection limit, because of a small NW volume. In order to confirm formation of the $\mathrm{V}_{\mathrm{Zn}}$ defects in the studied NWs, we have resorted to the ODMR technique that has a significantly higher sensitivity due to optical detection. ${ }^{[27]}$ Strong ODMR signals from the axial and non-axial $\mathrm{V}_{\mathrm{Zn}^{-}}$centers were indeed detected ${ }^{[7,28]}$ when monitoring visible light emissions, providing an unambiguous experimental proof for the formation of the zinc vacancies in the studied $\mathrm{ZnO}$ NWs. Together with the observed threshold of the TS-TPA upconversion process that coincides with the onset of the photon-ionization of the $\mathrm{V}_{\mathrm{Zn}}{ }^{2-}$, i.e. 
the (2-/-) level of $\mathrm{V}_{\mathrm{Zn}}$, this allows us to conclude that the $\mathrm{V}_{\mathrm{Zn}}$ defects also plays a dominant role in the energy upconversion in the $\mathrm{ZnO}$ NWs.

\section{Conclusion}

In summary we have demonstrated that defects commonly present in bulk and nanostructured $\mathrm{ZnO}$ can in fact extend functionality of this material as they can mediate efficient energy upconversion via the TS-TPA process. We have accurately determined the energy threshold of the process as being $2.10-2.14 \mathrm{eV}$. This threshold is found to be practically identical for all investigated materials irrespective of the employed growth techniques, which suggests that the intermediate state involved in the energy upconversion belongs to the same defect. Using photo-EPR we show that among all common contaminants and intrinsic defects, only the zinc vacancy has the ionization energy that closely matches the TS-TPA onset. The presence of this defect is also revealed in all materials that exhibit the TS-TPA. The obtained results thus provide compelling evidence that the zinc vacancy is the dominant defect responsible for the observed energy upconversion in bulk and nanostructured $\mathrm{ZnO}$. This work shows that the defect-mediated TS-TPA process can turn $\mathrm{ZnO}$ into an efficient energy-upconversion material without resorting to high photon densities, which can be exploited for a variety of applications in optoelectronics and photovoltaics. By identifying the chemical nature and the energy level of the defect responsible for TS-TPA, our finding also provides a useful guideline for further defect engineering of the energy upconversion efficiency by purposely incorporating the zinc vacancy defect, or by removing it if the associated energy upconversion process will be found detrimental to device applications for transparent electronics. 


\section{Experimental Section}

All structures investigated in this work were intentionally undoped. Bulk meltgrown $\mathrm{ZnO}$ single crystals from Cermet Inc. were n-type conductive with a carrier concentration ranging between $1 \times 10^{16}$ and $2 \times 10^{17} \mathrm{~cm}^{-3}$. The electron concentration was lower in hydrothermally-grown $\mathrm{ZnO}$ from Tokyo Denpa Co, i.e. around $5-9 \times 10^{15} \mathrm{~cm}^{-3}$. The $\mathrm{ZnO}$ NWs were grown on $\mathrm{Au}$ coated sapphire substrates using rapid thermal chemical vapor deposition (RTCVD) at $950{ }^{\circ} \mathrm{C}$. The growth was performed at a pressure of 20 Torr under the Ar and $\mathrm{O}_{2}$ flow as described in detail in Ref. 29. All samples have superior optical quality evident from the predominance of the BX emissions and only a weak (about $5-10 \%$ ) contribution of defect-related emissions in PL spectra within the visible spectral range.

The anti-stokes (or upconverted) PL was excited by using either second-harmonic pulses $\left(\lambda_{\text {exc }}=350-450 \mathrm{~nm}\right)$ of a tunable mode-locked Ti:sapphire solid state laser, with a pulse duration of 2 ps and a repetition rate of $76 \mathrm{MHz}$, or a dye laser tunable within the $575-$ $700 \mathrm{~nm}$ spectral range. The $266 \mathrm{~nm}$ line from a solid-state laser was used for the one-photon excitation above the bandgap energy. The incident laser beam was focused to a spot diameter of $\sim 0.5 \mathrm{~mm}$. The PL signals were detected at $5 \mathrm{~K}$ either by a photomultiplier tube assembled with a $0.8 \mathrm{~m}$ double-grating monochromator or by a streak camera combined with a $0.5 \mathrm{~m}$ single-grating monochromator. EPR measurements were carried out at temperatures between 4.2 and $77 \mathrm{~K}$ with a microwave frequency of $\sim 9 \mathrm{GHz}$. For photo-EPR investigations the same tunable dye laser as the one employed in the PL experiments was used. Before each photoEPR measurement, the sample was cooled down in dark from room temperature to $77 \mathrm{~K}$ prior to 3 min light illumination with a given photon energy. This was done to ensure the same initial condition for each measurement and also to avoid effects of ambient light and photoinduced recharging from preceding measurements. ODMR studies were performed at $3 \mathrm{~K}$ with a microwave (MW) frequency of $\sim 9 \mathrm{GHz}$ using the $364 \mathrm{~nm}$ line of an $\mathrm{Ar}^{+}$ion laser as an 
excitation source. ODMR spectra were obtained as a change of the integrated PL intensity detected by a Si detector within the visible spectral range of $420-1000 \mathrm{~nm}$.

\section{Supporting Information}

Supporting Information is available from the Wiley Online Library or from the author.

\section{Acknowledgements}

The financial support of this work by the Swedish Research Council (grant \# 621-2010-3971) is greatly appreciated.

Received: ((will be filled in by the editorial staff))

Revised: ((will be filled in by the editorial staff)) Published online: ((will be filled in by the editorial staff))

[1] A. Janotti and C. G. Van de Walle, Rep. Prog. Phys. 2009, 72, 126501.

[2] a) S. J. Pearton, D. P. Norton, M. P. Ivill, A. F. Hebard, J. M. Zavada, W. M. Chen and I. A. Buyanova, IEEE Transactions on Electron Devices 2007, 54, 1040; b) C. M. Lieber and Z. L. Wang, MRS Bulletin 2007, 22, 99.

[3] a) M. H. Huang, S. Mao, H. Feick, H. Yan, Y. Wu, H. Kind, E. Weber, R. Russo, and P. Yang, Science 2001, 292, 1897; b) M. Law, L. E. Greene, J. C. Johnson, R. Saykally, and P. Yang, Nat. Mater. 2005, 4, 455; c) J. J. Cole, X. Wang, R. J. Knuesel, and H. O. Jacobs, Nano Lett. 2008, 8, 1477.

[4] a) Y. L. Wu, C. S. Lim, S. Fu, A. I. Y. Tok, H. M. Lau, F. Y. C. Boey and X. T. Zeng, Nanotechnology 2007, 18, 215604; b) H.-M. Xiong, Adv. Mater. 2013, 25, 5329.

[5] C. Zhang, F. Zhang, T. Xia, N. Kumar, J. Hahm, J. Liu, Z. L. Wang, and J. Xu, Opt. Express 2009, 17, 7893.

[6] a) Y. C. Zhong, K. S.Wong, A. B. Djurišic, and Y. F. Hsu, Appl. Phys. B 2009, 97, 125; b) C. F. Zhang, Z. W. Dong, G. J. You, R. Y. Zhu, S. X. Qian, H. Deng, H. Cheng and J. C. Wang, Appl. Phys. Lett. 2006, 89, 042117; c) W. Cao, W. Du, F. Su, and G. Li, Appl. Phys. Lett. 2006, 89, 031902; d) S. Mani, J. I. Jang, and J. B. Ketterson, Appl. Phys. Lett. 2008, 93, 041902; e) S. K. Das, M. Biswas, D. Byrne, M. Bock, E. McGlynn, M. Breusing, and R. Grunwald, J. Appl. Phys. 2010, 108, 043107.

[7] S. L. Chen, J. Stehr, N. K. Reddy, C. W. Tu, W. M. Chen, and I. A. Buyanova, Appl. Phys. B 2012, 108, 919.

[8] F. Auzel, Chem. Rev. 2004, 104, 139.

[9] a) F. van de Rijke, H. Zijlmans, S. Li, T. Vail, A. K. Raap, R. S. Niedbala, and H. J. Tanke, Nature Biothechn. 2001, 19, 273; b) S. F. Lim, R. Riehn, W. S. Ryu, N. 
Khanarian, C. K. Tung, D. Tank, and R. H. Austin, Nano Lett. 2006, 6, 169; c) M. Nyk, R. Kumar, T. Y. Ohulchanskyy , E. J. Bergey and P. N. Prasad, Nano Lett. 2008, 8, 3834; d) B. E. Cohen, Nature 2010, 467, 407; e) F. Wang, Y. Han, C. S. Lim, Y. Lu, J. Wang, J. Xu, H. Chen, C. Zhang, M. Hong, and X. Liu, Nature 2010, 463, 1061.

[10] S. Sivakumar, F. C. J. M. van Veggel, and M. J. Raudsepp, J. Am. Chem. Soc. 2005, 127, 12464.

[11] S. L. Oliveira, D. S. Corrêa, L. Misoguti, C. J. L. Constantino, R. F. Aroca, S. C. Zilio, and C. R. Mendonça, Adv. Mater. 2005, 17, 1890.

[12] a) B. M. van der Ende, L. Aarts, and A. Meijerink, Phys. Chem. Chem. Phys. 2009, 11, 11081; b) G.-B. Shan and G. P. Demopoulos, Adv. Mater. 2010, 22, 4373; c) W. Zou, C. Visser, J. A. Maduro, M. S. Pshenichnikov, and J. C. Hummelen, Nat. Photonics 2012, 6,560 .

[13] D. A. Parthenopoulos and P. M. Rentzepis, Science 1989, 245, 843.

[14] E. Downing, L. Hesselink, J. Ralston, and R. Macfarlane, Science 1996, 273, 1185.

[15] F. Wang, R. Deng, J. Wang, Q. Wang, Y. Han, H. Zhu, X. Chen, and X. Liu, Nat. Mater. 2011, 10, 968 .

[16] V. A. Nikitenko, J. Appl. Spectrosc. 1992, 57, 783.

[17] J. E. Stehr, B. K. Meyer, and D. M. Hofmann, Appl. Magn. Reson. 2010, 39, 137.

[18] B. K. Meyer, H. Alves, D. M. Hofmann, W. Kriegseis, D. Forster, F. Bertram, J. Christen, A. Hoffmann, M. Straßburg, M. Dworzak, U. Haboeck, and A. V. Rodina, Phys. Status Solidi (b) 2004, 241, 231.

[19] S. L. Chen, W. M. Chen, and I. A. Buyanova, Phys. Rev. B 2012, 86, 235205.

[20] S. Stoll and A. Schweiger, J. Magn. Reson. 2006, 178, 42.

[21] P. Dorain, Phys. Rev. 1958, 112, 1058.

[22] a) J. M. Smith and W. E. Vehse, Phys. Lett. A 1970, 31, 147; b) C. Gonzalez, D. Galland, and A. Herve, Phys. Status Solidi (b) 1975, 72, 309.

[23] a) X. J. Wang, L. S. Vlasenko, S. J. Pearton, W. M. Chen, and I. A. Buyanova, J. Phys. D. Appl. Phys. 2009, 42, 175411; b) S. M. Evans, N. C. Giles, L. E. Halliburton, and L. A. Kappers, J. Appl. Phys. 2008, 103, 043710; c) R. Laiho, L. S. Vlasenko, and M. P. Vlasenko, J. Appl. Phys. 2008, 103, 123709.

[24] D. Galland and A. Herve, Phys. Lett. A 1970, 33, 1.

[25] P. H. Kasai, Phys. Rev 1963, 130, 989.

[26] M. D. McCluskey and S. J. Jokela, J. Appl. Phys. 2009, 106, 071101.

[27] W. M. Chen, Thin Solid Films 2000, 364, 45.

[28] J. E. Stehr, S. L. Chen, S. Filippov, M. Devika, N. Koteeswara Reddy, C. W. Tu, W. M. Chen, and I. A. Buyanova, Nanotechnology 2013, 24, 015701.

[29] Q. J. Ren, S. Filippov, S. L. Chen, M. Devika, N. Koteeswara Reddy, C. W. Tu, W. M. Chen, and I. A. Buyanova, Nanotechnology 2012, 23, 425201. 


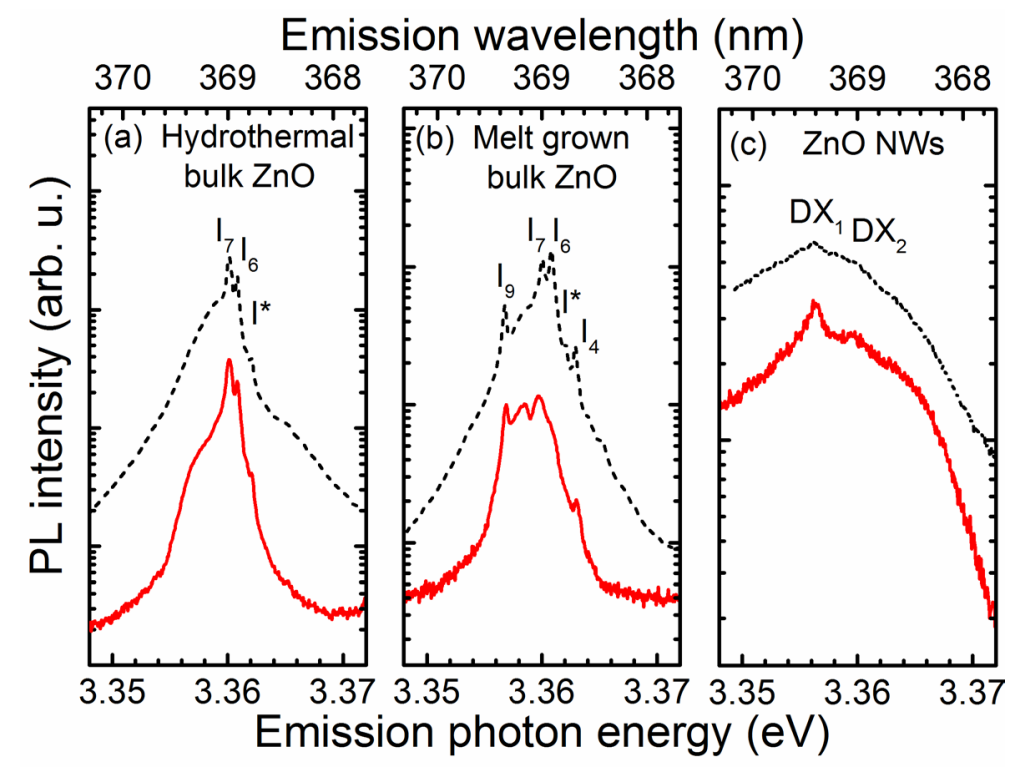

Figure 1. Representative PL spectra measured within the excitonic spectral range from bulk hydrothermal (a) and melt-grown (b) $\mathrm{ZnO}$ and also from $\mathrm{ZnO}$ NWs (c). The solid (red online) and dashed (black online) lines represent spectra measured under two-photon $\left(\lambda_{\text {exc }}=400 \mathrm{~nm}\right)$ and one-photon $\left(\lambda_{\mathrm{exc}}=266 \mathrm{~nm}\right)$ excitation, respectively. The spectra are displayed in the semi-logarithmic scale and are offset vertically, for clarity.

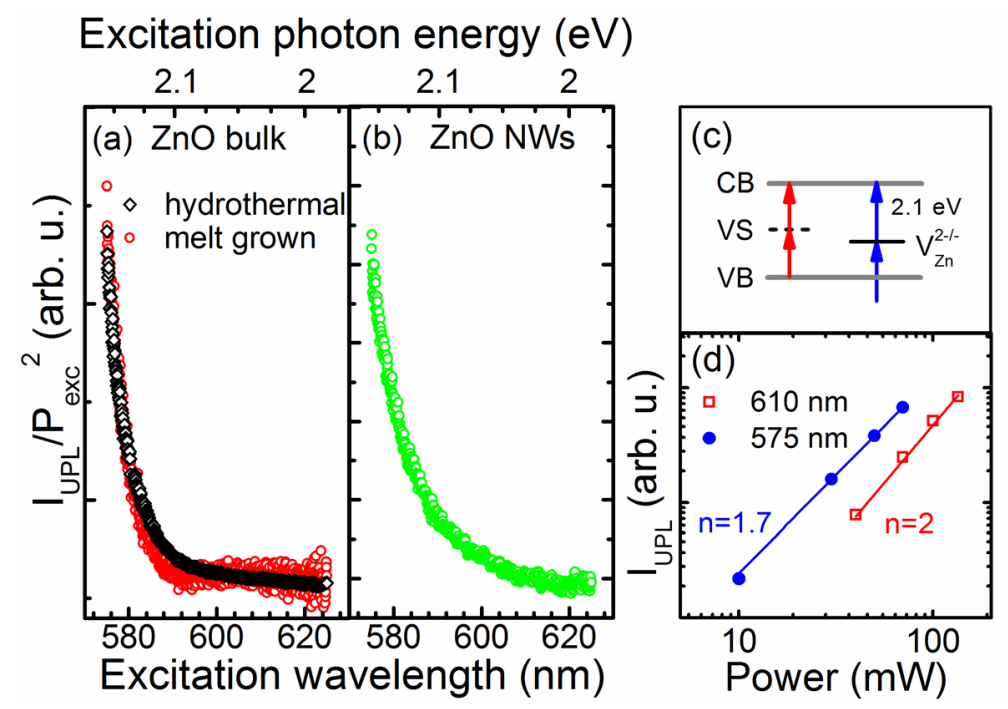

Figure 2. PLE spectra measured at $5 \mathrm{~K}$ by monitoring the dominant UPL emission from the bulk (a) and NWs (b) samples. (c) Schematic picture of the TPA and TS-TPA processes via virtual and real defect (zinc vacancy) states, which are labeled as VS and ( $\left.\mathrm{V}_{\mathrm{Zn}}\right)$, respectively. (d) Excitation power dependences of the UPL emission measured from the bulk $\mathrm{ZnO}$ with $\lambda_{\text {exc }}$ $=575 \mathrm{~nm}$ (the solid dots) and $610 \mathrm{~nm}$ (open squares). The data are displayed in a doublelogarithmic scale. The solid lines represent fits by the relation $\mathrm{I}_{\mathrm{UPL}} \sim \mathrm{P}^{\mathrm{n}}{ }_{\text {exc }}$ with the specified $\mathrm{n}$. 


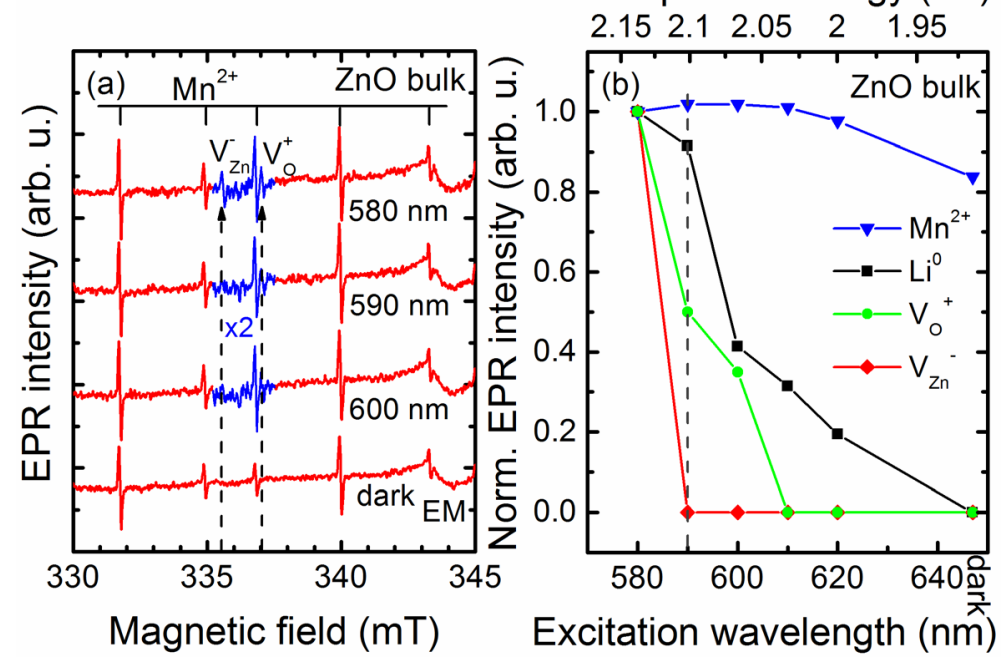

Figure 3. (a) EPR spectra from the melt-grown $\mathrm{ZnO}$ measured at $77 \mathrm{~K}$ with a microwave frequency of $9.4 \mathrm{GHz}$ in dark and under light illumination. An applied magnetic field was oriented parallel to the c-axis of the $\mathrm{ZnO}$ crystal. (b) Intensities of the detected EPR signals as a function of excitation wavelength. All data are normalized to the same peak intensity. The dashed line represents the spectral threshold for the TS-TPA process deduced from the PLE measurements of the UPL emission.

Table 1. Summary of the spin-Hamiltonian parameters of the defects discussed in this work. The axial components of the electron $\mathbf{g}$-tensor are denoted as $\mathrm{g}_{\perp}$ and $\mathrm{g} \|$, while the components for the non-axial centers are given by $g_{x x}, g_{y y}$ and $g_{z z}$. For the non-axial centers, $\varphi$ is the angle between the $z$ and $c$ axis. The perpendicular and parallel components $\mathrm{A}_{\perp}$ and $\mathrm{A} \|$ of the hyperfine interaction tensor $\mathbf{A}$ and the fine structure parameter $\mathrm{D}$ are given in $\mathrm{MHz}$. With $\mathrm{D}_{\mathrm{zz}}$ $=2 \mathrm{D} / 3$ and $\mathrm{D}_{\mathrm{xx}}=\mathrm{D}_{\mathrm{yy}}=-\mathrm{D} / 3$. The parallel and perpendicular directions are with respect to the $c$-axis.

\begin{tabular}{|c|c|c|c|c|c|c|c|c|}
\hline Center & $\mathrm{S}$ & I & $\begin{array}{lll}g_{x x} & \left(g_{\perp}\right) \quad g_{y y}\end{array}$ & $\mathrm{~g}_{\mathrm{zz}}(\mathrm{g} \|)$ & $\left|\mathrm{A}_{\perp}\right|$ & $|\mathrm{A} \||$ & $\mathrm{D}$ & $\varphi(\operatorname{deg})$ \\
\hline $\left.\mathrm{V}_{\mathrm{Zn}^{-}}{ }^{(\text {axial }}\right)$ & $1 / 2$ & & 2.0193 & 2.0041 & & & & \\
\hline $\mathrm{V}_{\mathrm{Zn}}{ }^{-}($non-axial $)$ & $1 / 2$ & & $2.0173 \quad 2.0183$ & 2.0041 & & & & 110.75 \\
\hline $\mathrm{Li}^{0}$ (axial) & $1 / 2$ & $3 / 2$ & 2.0253 & 2.0028 & 5.12 & 0.61 & & \\
\hline $\mathrm{Li}^{0}$ (non-axial) & $1 / 2$ & $3 / 2$ & $2.0223 \quad 2.0254$ & 2.0040 & 5.1 & 0.81 & & 3.1 \\
\hline $\mathrm{VO}^{+}$ & $1 / 2$ & & 1.9960 & 1.9945 & & & & \\
\hline EM & $1 / 2$ & & 1.955 & 1.957 & & & & \\
\hline $\mathrm{Fe}^{3+}$ & $5 / 2$ & $1 / 2$ & 2.0060 & 2.0060 & 27.04 & 27.04 & -1779.9 & \\
\hline $\mathrm{Mn}^{2+}$ & $5 / 2$ & $5 / 2$ & 2.0016 & 2.0016 & 227.8 & 227.8 & -650.2 & \\
\hline
\end{tabular}

